\title{
Effectiveness of Mindfulness Intervention on Cognitive Functions: A Meta-analysis of Mindfulness Studies
}

\section{Efectividad de la intervención de Mindfulness en funciones cognitivas: un metaanálisis de estudios de Mindfulness}

\section{Marzieh Nazaribadie}

Research Center for Behavioral Disorders and Substance Abuse, Hamadan University of Medical Sciences, Hamadan, Iran.

ORCID: 0000-0002-9369-6996

\section{Ali Ghaleiha}

Research Center for Behavioral Disorders and Substance Abuse, Hamadan University of

Medical Sciences, Hamadan, Iran.

ORCID: 0000-0003-3037-2676

Mohammad Ahmadpanah

Research Center for Behavioral Disorders and Substance Abuse, Hamadan University of

Medical Sciences, Hamadan, Iran.

ORCID: 0000-0003-2908-2460

\section{Mehrdokht Mazdeh}

Research Center for Behavioral Disorders and Substance Abuse, Hamadan University of Medical Sciences, Hamadan, Iran.

ORCID: 0000-0001-6253-5850

\section{Nasrin Matinnia}

Nursing department, Hamedan branch, Islamic Azad University, Hamedan, Iran.

ORCID: 0000-0001-9526-9974

Mohammad-Kazem Zarabian

Research Center for Behavioral Disorders and Substance Abuse, Hamadan University of

Medical Sciences, Hamadan, Iran.

ORCID: 0000-0001-6369-3033

\section{Negar Ostovar}

Department of Education, Payam Noor Uiversity, Bostan Abad Campus, Iran. ORCID: 0000-0003-4853-3298 


\section{Summary}

Background: The experience of cognitive deficits is common among patients with degenerative and psychiatric disorders. Objective: The present study aimed to evaluate the effect of the empirical literature of mindfulness intervention on cognitive functions. Methods: This study was conducted in June 2020 by using the scientific records were retrieved by a systematic search of several bibliographic databases on the Medline, Scopus, ISI Web of Sciences, Google scholar database from 2000 to 2020 for testing the effect of mindfulness intervention on cognitive functions. For data analysis CMA2 software were used. Heterogeneity assessed by Cochran's Q statistics test. Publication Bias assessed by Orwin fail safe N, Begg's method kendall's Tau, Egger's method intercept and funnel plot. Results: from 17 initial studies, 28 effect sizes were calculated. Among the 28 effect sizes, 6 indicators were negative and 22 indicators were positive. key results from the meta-analysis, Compared to healthy controls showed that people were receive mindfulness intervention significantly improved in working memory and attention function, with this enhance medium magnitude (Hedges' $g=0.32,0.35$ respectively, see Figure 2). There was no significant improve by use mindfulness intervention on executive function. Conclusions: The results prove the initial evidence that mindfulness intervention can improve some neurocognitive processing such as attention function and working memory

Keywords: Mindfulness; Cognitive Functions; Meta-Analysis.

\section{Resumen}

Antecedentes: la experiencia de los déficits cognitivos es común entre los pacientes con trastornos degenerativos y psiquiátricos. Objetivo: El presente estudio tuvo como objetivo evaluar el efecto de la literatura empírica de la intervención de mindfulness sobre las funciones cognitivas. Métodos: Este estudio se realizó en junio de 2020 mediante el uso de registros científicos que se recuperaron mediante una búsqueda sistemática de varias bases de datos bibliográficas en Medline, Scopus, ISI Web of Sciences, base de datos académica de Google de 2000 a 2020 para probar el efecto de la intervención de mindfulness en funciones cognitivas. Para el análisis de datos se utilizó el software CMA2. Heterogeneidad evaluada por la prueba de estadística Q de Cochran. Sesgo de publicación evaluado por Orwin a prueba de fallos N, método de Begg, Tau de Kendall, método de intercepción y gráfico de embudo de Egger. Resultados: de 17 estudios iniciales, se calcularon 28 tamaños de efecto. Entre los 28 tamaños de efecto, 6 indicadores fueron negativos y 22 indicadores fueron positivos. Los resultados clave del metanálisis, en comparación con los controles sanos, mostraron que las personas que recibieron la intervención de atención plena mejoraron significativamente en la memoria de trabajo y la función de atención, con esta mejora de magnitud media (g de Hedges $=0.32,0.35$ respectivamente, ver Figura 2). No hubo una mejora significativa mediante el uso de la intervención de atención plena en la función ejecutiva. Conclusiones: Los resultados prueban la evidencia inicial de que la intervención de mindfulness puede mejorar algunos procesos neurocognitivos como la función de atención y la memoria de trabajo.

Palabras llave: Mindfulness; Funciones cognitivas; Metaanálisis

\section{Introduction}

Mindfulness is a therapy proposed by Kabat-Zinn(1). Mindfulness is the understanding of what is happening within us as well as in the environment around us at the present time and without any judgment, and it is a tool that helps us manage our thoughts and feelings(2) Mindfulness is a kind of internal consciousness regarding automated processes and unintentional processing of internal currents and events, especially in thoughts. In this situation, individuals learn to 
cognitively separate themselves from their thoughts(3). Mindfulness can regulate choice of diverse contemplate and behavioral ways, and further use of mindfulness strengthens the new design to regulate the neurocognitive processing of the mind(4). Cognition is a means of insight into the world around us, and we use knowledge gained through cognition in our decisions to act purposefully(5). Cognition primarily refers to things like memory, the ability to learn new information, speech, reading, and comprehension(6). Cognitive functions refer to a person's ability to process thoughts, the most important cognitive domains in neuropsychological assessments include: memory, attention and executive functions(6). There have been several pathways leading to clinical implementations of mindfulness practices $(3,4,7-14)$. Much research has been done on mindfulness as an integrated mind exercise, and most accumulated research shows a significant improvement in emotion regulation by using mindfulness. (15). In a general view, meta-analytic studies have concluded that mindfulness is moderately effective in reducing anxiety and depression(16). In recent years, many studies have examined the effect of mindfulness on neuropsychological functions of the mind (17-22), and many studies in clinical works used various meditation such as (Mindfulness Based Stress Reduction (MBSR), Mindfulness-Based Cognitive Therapy (MBCT), etc ) on cognitive functions(19, 20, 23-25).

2. Objective: Although meta-analytic studies on the effects of mindfulness on cognition are controversial and generally still under investigation(21), the aim of this meta-analysis is to summarize the effects of mindfulness on cognitive functions.

\section{Methods \\ Research Procedure}

This study was conducted in June 2020 by using the scientific records were retrieved by a systematic search of several bibliographic databases on the Medline, Scopus, Web of Sciences, Google scholar database from 2000 to 2020 and additional resources were searched using the set terms "mindfulness", "cognitive function", "cognitive performance", "cognitive processing", "executive function", "working memory function, attention function, executive performance, working memory performance, attention performance", "working memory function" and "working memory capacity". The references of the selected documents were also extracted to identify further qualified studies to be covered in the whole analysis. Furthermore, complete search for more grey literatures was done on Google. Studies evaluated of the English language database until June 2020.

\section{Inclusion and exclusion criteria}

In this research, studies were used that examined the effect of mindfulness on cognitive functions, and most of the studies were random controlled trial with pre/post-test in experimental and control groups. The studies were used that include sub groups of mindfulness such as Mindfulness Based Stress Reduction (MBSR), Mindfulness-Based Cognitive Therapy (MBCT), Mindfulness Awareness Program (MAPs), Detached Mindfulness (DM), Metacognitive model of Detached Mindfulness (MDM) and Learning to BREATHE (L2B). A number of studies that did not have enough data to calculate the effect size were removed from the effect size in the analysis process. Studies analyzing multidimensional interventions and all off the studies that they were case reports, or did not mention mean and standard deviation or confidence interval were deleted from this study.

Table 1 contain all Characteristics of studies such as sample of study, study design, protocol, cognition functions domain scales were used in studies and study name(table1). 
Table 1: Characteristics of the studies Mindfulness intervention on neurocognitive processing

\begin{tabular}{|c|c|c|c|c|}
\hline study & $\begin{array}{l}\text { Sample of } \\
\text { study }\end{array}$ & $\begin{array}{c}\text { Study } \\
\text { design \& } \\
\text { protocol }\end{array}$ & $\begin{array}{c}\text { Cognition Function } \\
\text { domain\& scales were } \\
\text { used }\end{array}$ & Study name \\
\hline $\begin{array}{c}\text { Alfonso } \\
\text { J. et al, } \\
2011\end{array}$ & $\begin{array}{c}\text { community } \\
\text { treatment: } \\
(\mathrm{n}=18) ; \\
\text { Standard } \\
\text { Treatment } \\
\text { alone }(\mathrm{n}=16)\end{array}$ & $\begin{array}{c}\text { Not } \\
\text { specified \& } \\
(\mathrm{MF})\end{array}$ & $\begin{array}{l}\text { Executive Function: } \\
\text { Stroop Color Words. } \\
\text { Working memory: } \\
\text { Digit Span subtests } \\
\text { from the WAIS-III }\end{array}$ & $\begin{array}{l}\text { Combined goal } \\
\text { management training } \\
\text { and mindfulness } \\
\text { meditation improve } \\
\text { executive functions and } \\
\text { decision-making } \\
\text { performance in abstinent } \\
\text { poly substance } \\
\text { abusers(17). }\end{array}$ \\
\hline $\begin{array}{l}\text { Chamber } \\
\text { s R. et a, } \\
2007\end{array}$ & $\begin{array}{l}\text { Mindfulness } \\
(\mathrm{n}=20) ; \\
\text { Control }(\mathrm{n}= \\
20)\end{array}$ & $\begin{array}{l}\text { Active } \\
\text { (opt-in) } \\
\text { recruitment } \\
\text { procedures } \\
\& \\
\text { (IMM) }\end{array}$ & $\begin{array}{c}\text { Attention: Internal } \\
\text { Switching Task } \\
\text { Working memory: } \\
\text { DSB= Digit Span } \\
\text { Backward (subscale } \\
\text { of the Wechsler Adult } \\
\text { Intelligence Scale, 3rd } \\
\text { Edition; WAIS III) }\end{array}$ & $\begin{array}{l}\text { The Impact of Intensive } \\
\text { Mindfulness Training on } \\
\text { Attention Control, } \\
\text { Cognitive Style, and } \\
\text { Affect(35). }\end{array}$ \\
\hline $\begin{array}{l}\text { Ching. H. } \\
\text { et al, } \\
2015\end{array}$ & $\begin{array}{l}\text { Intervention: } \\
(n=112) \\
\text { control: } \\
(n=66)\end{array}$ & $\begin{array}{c}\text { Quasi- } \\
\text { experiment } \\
\text { al } \\
\text { Pre/post- } \\
\text { test design } \\
\& \\
\text { (MMC) } \\
\end{array}$ & $\begin{array}{l}\text { Attention : Digit } \\
\text { vigilance task } \\
\text { Accuracy } \\
\text { Working memory: } \\
\text { Spatial working } \\
\text { memory } \\
\text { Accuracy }\end{array}$ & $\begin{array}{l}\text { Effects of a Mindfulness } \\
\text { Meditation Course on } \\
\text { Learning and Cognitive } \\
\text { Performance among } \\
\text { University Students in } \\
\text { Taiwan(18) }\end{array}$ \\
\hline $\begin{array}{c}\text { Flook } \\
\text { L,et al. } \\
2010\end{array}$ & $\begin{array}{l}\text { MAPs }(n= \\
\text { 32); control: } \\
\quad(n=23)\end{array}$ & $\begin{array}{c}\text { Not } \\
\text { Specified } \\
\& \\
\text { (MAPs) }\end{array}$ & $\begin{array}{l}\text { Executive Function: } \\
\text { Behavior Rating } \\
\text { Inventory of } \\
\text { Executive Function } \\
\text { (BRIEF) }\end{array}$ & $\begin{array}{c}\text { Effects of Mindful } \\
\text { Awareness Practices on } \\
\text { Executive Functions in } \\
\text { Elementary School } \\
\text { Children(36). }\end{array}$ \\
\hline $\begin{array}{c}\text { Jerman F } \\
\text { et al, } \\
2013\end{array}$ & $\begin{array}{l}\operatorname{MBCT}(n=20) ; \\
\operatorname{Control}(n=20)\end{array}$ & $\begin{array}{c}\text { Randomize } \\
\text { d Control } \\
\text { trial (RCT) } \\
\& \\
\text { (MBCT) }\end{array}$ & $\begin{array}{l}\text { Executive Function: } \\
\text { Plus-Minus (PM) }\end{array}$ & $\begin{array}{c}\text { Cognitive Functioning in } \\
\text { Patients Remitted from } \\
\text { Recurrent Depression: } \\
\text { Comparison with } \\
\text { Acutely Depressed } \\
\text { Patients and Controls } \\
\text { and Follow-up of a } \\
\text { Mindfulness-Based } \\
\text { Cognitive Therapy } \\
\text { Trial(23). }\end{array}$ \\
\hline $\begin{array}{c}\text { Johns } \\
\text { SA, et al } \\
2016\end{array}$ & $\begin{array}{l}\text { MBSR } n=35 \\
{ }^{* 1} \mathrm{ES} \mathrm{n}=36\end{array}$ & $\begin{array}{l}\text { Randomize } \\
\mathrm{d} \\
\text { controlled } \\
\text { pilot trial } \\
\& \text { (MBSR) }\end{array}$ & $\begin{array}{l}\text { Executive Function: } \\
\text { Stroop color-word test } \\
\text { Attention Function: } \\
\text { The Attention } \\
\text { Function Index (AFI) }\end{array}$ & $\begin{array}{l}\text { Randomized controlled } \\
\text { pilot trial of } \\
\text { mindfulness-based stress } \\
\text { reduction for breast and } \\
\text { colorectal cancer } \\
\text { survivors: effects on } \\
\text { cancer-related cognitive } \\
\text { impairment }(19) \text {. }\end{array}$ \\
\hline
\end{tabular}




\begin{tabular}{|c|c|c|c|c|}
\hline $\begin{array}{l}\text { kanei. L } \\
\text { et al } \\
2019\end{array}$ & $\begin{array}{l}\text { N( L2B:N=53 } \\
*^{2} \text { IAU:N=62) }\end{array}$ & $\begin{array}{l}\text { Controlled- } \\
\text { trial study } \\
\& \text { (L2B). }\end{array}$ & $\begin{array}{c}\text { Attention: YSR, } \\
\text { Youth Self-Report (29 } \\
\text { items): Attention, } \\
\text { Attention Syndrome } \\
\text { subscale } \\
\text { Executive Function: } \\
\text { Self-Report version } \\
\text { (BRIEF-SR; Guy } \\
\text { et al. 2004) }\end{array}$ & $\begin{array}{c}\text { Effects of a Brief } \\
\text { Mindfulness Curriculum } \\
\text { on Self-reported } \\
\text { Executive Functioning } \\
\text { and Emotion Regulation } \\
\text { in Hong Kong } \\
\text { Adolescent(20). }\end{array}$ \\
\hline $\begin{array}{l}\text { Klainin- } \\
\text { Yobas P } \\
\text { et al, } \\
2019\end{array}$ & $\begin{array}{l}\text { Mindfulness } \\
\text { Awareness } \\
\text { Intervention } \\
\text { (MAP) } \\
(\mathrm{n}=28), \\
\text { Health } \\
\text { Education } \\
\text { Program } \\
\text { (HEP) } \\
(\mathrm{n}=27)\end{array}$ & $\begin{array}{l}\text { A } \\
\text { randomize } \\
\quad \mathrm{d} \\
\text { controlled } \\
\text { trial \& } \\
\text { (MAPs) }\end{array}$ & $\begin{array}{l}\text { working memory: } \\
\text { Clinical } \\
\text { Dementia Rating } \\
\text { (CDR) }\end{array}$ & $\begin{array}{l}\text { The effects of } \\
\text { mindfulness and health } \\
\text { education programs on } \\
\text { the emotional state and } \\
\text { cognitive function of } \\
\text { elderly individuals with } \\
\text { mild cognitive } \\
\text { impairment: A } \\
\text { randomized controlled } \\
\text { trial( } 24) \text {. }\end{array}$ \\
\hline $\begin{array}{l}\text { Luu. K, } \\
\text { et al, } \\
2017\end{array}$ & $\begin{array}{l}\text { Meditation } \\
\quad(\mathrm{n}=31) \\
\text { Control } \\
(\mathrm{n}=31)\end{array}$ & $\begin{array}{l}\text { Not } \\
\text { specified \& } \\
\text { (MBSR) }\end{array}$ & $\begin{array}{l}\text { Executive Function: } \\
\text { Stroop task }\end{array}$ & $\begin{array}{l}\text { Examining the Acute } \\
\text { Effects of Hatha Yoga } \\
\text { and Mindfulness } \\
\text { Meditation on Executive } \\
\text { Function and Mood(21). }\end{array}$ \\
\hline $\begin{array}{l}\text { Noone C, } \\
\text { et al } \\
2018\end{array}$ & $\begin{array}{c}\text { Sham } \\
\text { Meditation( }= \\
48) ; \\
\text { Mindfulness } \\
\text { meditation(n= } \\
\text { 43) }\end{array}$ & $\begin{array}{l}\text { randomize } \\
\text { d active- } \\
\text { controlled } \\
\text { trial \& } \\
\text { (MM) }\end{array}$ & $\begin{array}{l}\text { Executive Function: } \\
\text { Sternberg executive } \\
\text { control task }\end{array}$ & $\begin{array}{l}\text { A Randomized active- } \\
\text { controlled trial to } \\
\text { examine the effects of an } \\
\text { online mindfulness } \\
\text { intervention on } \\
\text { executive control, } \\
\text { critical thinking and key } \\
\text { thinking dispositions in a } \\
\text { university student } \\
\text { sample(22). }\end{array}$ \\
\hline $\begin{array}{l}\text { Quach D, } \\
\text { et al, } \\
2015\end{array}$ & $\begin{array}{l}\text { Mindfulness } \\
\text { meditation( } \mathrm{n}= \\
\text { 54); Waitlist } \\
\text { analyzed }(n= \\
\text { 53)) }\end{array}$ & $\begin{array}{l}\text { randomize } \\
\quad \mathrm{d} \\
\text { controlled } \\
\text { trial \& } \\
\text { (MBCT) }\end{array}$ & $\begin{array}{c}\text { Working memory: } \\
\text { Automated Operation } \\
\text { Span } \\
\text { Task (AOSPAN) }\end{array}$ & $\begin{array}{c}\text { A Randomized } \\
\text { Controlled Trial } \\
\text { Examining the Effect of } \\
\text { Mindfulness Meditation } \\
\text { on Working Memory } \\
\text { Capacity in } \\
\text { Adolescents(37). }\end{array}$ \\
\hline $\begin{array}{l}\text { Shapero } \\
\text { B, et al } \\
2018\end{array}$ & $\begin{array}{c}\text { MBCT } \\
\text { program }(\mathrm{N}= \\
\text { 22) or waitlist } \\
(\mathrm{N}=18) .\end{array}$ & $\begin{array}{l}\text { randomize } \\
\text { d } \\
\text { controlled } \\
\text { trial \& } \\
\text { (MBCT) }\end{array}$ & $\begin{array}{l}\text { Executive function: } \\
\text { Cognitive Flexibility } \\
\text { Inventory (CFI) }\end{array}$ & $\begin{array}{l}\text { Mindfulness-Based } \\
\text { Cognitive Therapy } \\
\text { Improves Cognitive } \\
\text { Functioning and } \\
\text { Flexibility Among } \\
\text { Individuals with } \\
\text { Elevated Depressive } \\
\text { Symptoms }(38) .\end{array}$ \\
\hline $\begin{array}{c}\text { Tang R, } \\
\text { et al } \\
2020 \\
\end{array}$ & $\begin{array}{l}\text { Mindfulness: } \\
\quad(\mathrm{n}=37)\end{array}$ & $\begin{array}{c}\text { Not } \\
\text { specified \& } \\
\text { (MBSR) }\end{array}$ & $\begin{array}{l}\text { Executive function: } \\
\text { Attention Network } \\
\text { Test (ANT) } \\
\end{array}$ & $\begin{array}{l}\text { A College First-Year } \\
\text { Mindfulness Seminar to } \\
\text { Enhance Psychological }\end{array}$ \\
\hline
\end{tabular}




\begin{tabular}{|c|c|c|c|c|}
\hline & $\begin{array}{c}\text { Psychology:(n } \\
=14)\end{array}$ & & $\begin{array}{c}\text { Working memory: } \\
\text { Operation Span Task } \\
\text { (OSPAN) } \\
\text { Attention: Sustained } \\
\text { Attention to Response } \\
\text { Task (SART) }\end{array}$ & $\begin{array}{c}\text { Well-Being and } \\
\text { Cognitive Function(39). }\end{array}$ \\
\hline $\begin{array}{l}\text { Thierry } \\
\text { K, et al } \\
2018\end{array}$ & $\begin{array}{l}\text { Mindfulness(n } \\
=157) \\
\text { Control(n= } \\
139)\end{array}$ & $\begin{array}{c}\text { Not } \\
\text { Specified } \\
\& \\
(\mathrm{MBCT})\end{array}$ & $\begin{array}{l}\text { Executive function: } \\
\text { flanker fish task }\end{array}$ & $\begin{array}{c}\text { A Self-Oriented } \\
\text { Mindfulness-Based } \\
\text { Curriculum Improves } \\
\text { Prekindergarten } \\
\text { Students' Executive } \\
\text { Functions }(40) .\end{array}$ \\
\hline $\begin{array}{l}\text { Valls- } \\
\text { Serrano } \\
\text { C, } 2016\end{array}$ & $\begin{array}{l}\text { MM group }(n \\
=15) ; \\
\text { 3*TAU }(n= \\
15)\end{array}$ & $\begin{array}{c}\text { Not } \\
\text { Specified } \\
\& \text { (MBSR) }\end{array}$ & $\begin{array}{c}\text { Executive: Color- } \\
\text { word interference test } \\
\text { Stroop } \\
\text { Working memory: } \\
\text { Letter-number } \\
\text { Sequencing of WAIS- } \\
\text { III }\end{array}$ & $\begin{array}{c}\text { Goal Management } \\
\text { Training + Mindfulness } \\
\text { Meditation improves } \\
\text { executive functions and } \\
\text { transfers to ecological } \\
\text { tasks of daily life in poly } \\
\text { substance users enrolled } \\
\text { in therapeutic } \\
\text { community } \\
\text { treatment }(41) .\end{array}$ \\
\hline $\begin{array}{c}\text { Wahbeh } \\
\text { H, et al } \\
2016\end{array}$ & $\begin{array}{l}\text { IMMI:(n=8); } \\
\text { Education }(n=)\end{array}$ & $\begin{array}{c}\text { pilot study } \\
\& \\
\text { (MBCT) }\end{array}$ & $\begin{array}{c}\text { Executive function: } \\
\text { Flanker Task Conflict } \\
\text { effect } \\
\text { Working memory: } \\
\text { Letter Number } \\
\text { Sequencing } \\
\end{array}$ & $\begin{array}{l}\text { Internet mindfulness } \\
\text { meditation for cognition } \\
\text { and mood in older } \\
\text { adults: a pilot study(42). }\end{array}$ \\
\hline $\begin{array}{l}\text { Wood L, } \\
\text { et al, } \\
2018\end{array}$ & $\begin{array}{c}\text { Intervention:( } \\
\mathrm{n}=10) \text {; wait- } \\
\text { list } \\
\text { Control:( } \mathrm{n}=11 \\
\text { ) }\end{array}$ & $\begin{array}{l}\text { randomize } \\
\quad \mathrm{d}, \\
\text { controlled } \\
\text { pilot study } \\
\& \text { (MBCT) }\end{array}$ & $\begin{array}{l}\text { Executive function: } \\
\text { Mini-Mind rating of } \\
\text { EF*4 } \\
\text { Working memory: } \\
\text { Mini-Mind rating of } \\
\text { EF } \\
\text { Attention: Mini-Mind } \\
\text { rating of EF }\end{array}$ & $\begin{array}{l}\text { Enhancing executive } \\
\text { function skills in } \\
\text { preschoolers through a } \\
\text { mindfulness-based } \\
\text { intervention: A } \\
\text { randomized, controlled } \\
\text { pilot study(43). }\end{array}$ \\
\hline
\end{tabular}

${ }^{* 1}$ education/support, ${ }^{* 2}$ intervention as usual, ${ }^{* 3}$ Treatment as usual; ${ }^{* 4}$ Executive function

\section{Data Analysis}

CMA2 software was used to calculate the effect sizes and sub sequent statistical analyzes related to the composition of the results. In this study, we use Hedges' $\mathbf{g}$ for assess effect size and heterogeneity was assessed by using Cochran's Q test. Fixed or random effect models were used to determine the composition according to the heterogeneity results. One of the main assumptions of the meta- analysis is publication bias. In this meta-analysis, a graphical diagram (funnel diagram) and statistical index (Fail-safe $\mathrm{N}$ ) were used to examine the publication bias. The random effects model was used as it better accommodates heterogeneous effect distributions

\section{Results}

Literature Search: The mean age of participants in articles used for review was 32, and 1310 articles were found in databases then un-related and duplicate articles were removed from the 
analysis and remain 35 articles in this section. At least 17full-text articles remained for metaanalysis (Figure1).

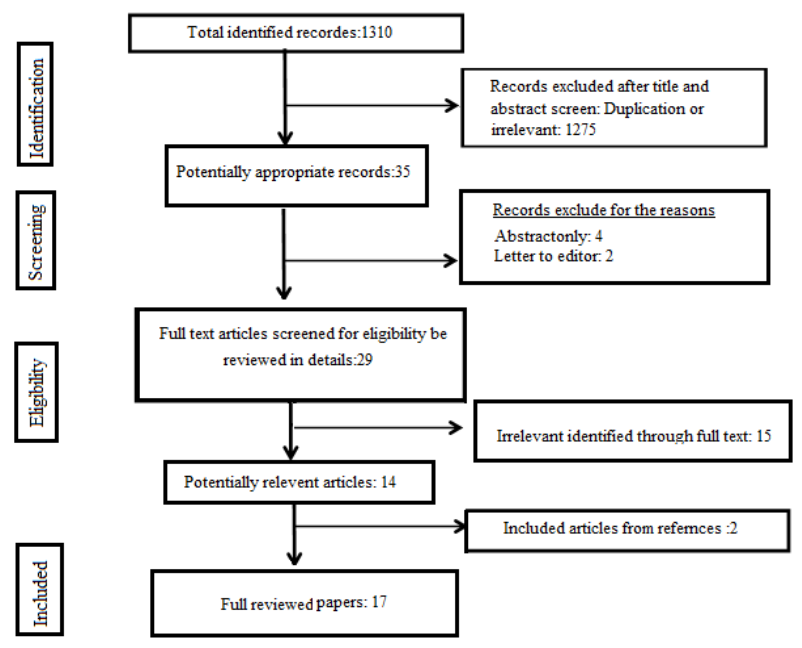

The PRISMA flowchart of the study design process

Participant Characteristics: As shown in figure 2, for 17 initial studies, 28 effect sizes were calculated. Among the 28 effect sizes, 6 indicators are negative and 22 indicators are positive. A negative effect of effect size indicates that mindfulness intervention has no effect on executive function. Examination of significant levels showed that, 20 effect sizes were not significant and 7 effect sizes were significant at the level of 0.01 or less, and the effect size of the 2 studies was significant at the level of 0.05 (Figure2).

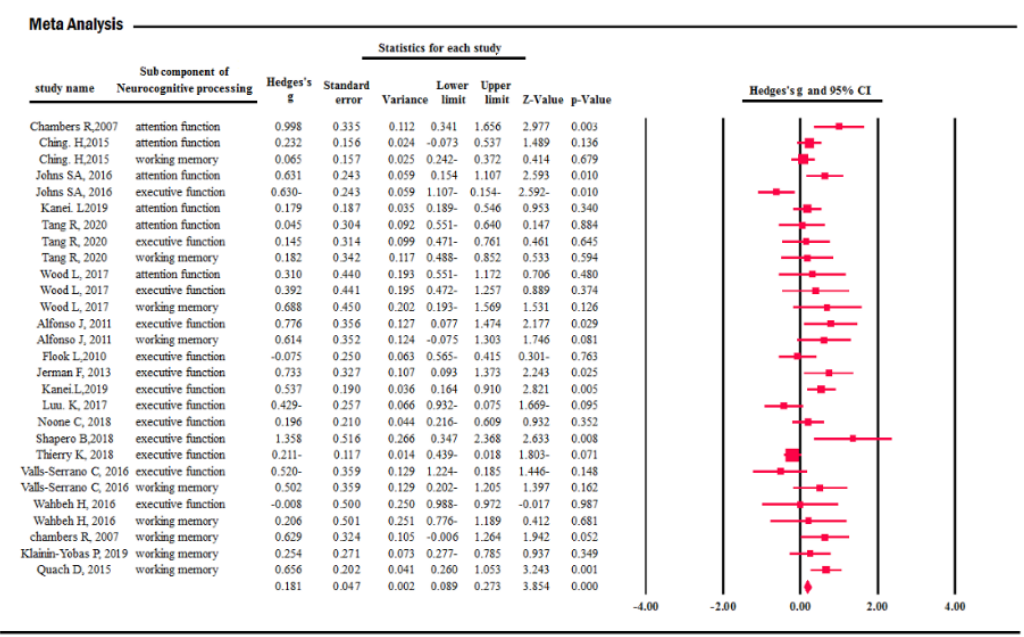

Table 2 reports the key results from the meta-analysis, Compared to healthy controls, people were receive mindfulness intervention significantly improved in working memory and attention function, with this enhance medium magnitude (Hedges' $\mathbf{g}=0.32,0.35$ respectively, see Figure 2). There was no significant improve by use mindfulness intervention in executive function. Cochran's Q used to ensure the heterogeneity of the initial research effect size, Fixed or random effect models were used to determine the composition according to the heterogeneity results. In this meta-analysis we report random effect for executive function, $\mathrm{M} / \mathrm{F}$ and $\mathrm{F}$.

Heterogeneity of Effect Sizes: As reported in Table 2, Cochran's Q statistics for some mean effects were significant, indicating that there is meaningful variance between the studies 
that are contributing to each mean subcomponent's mean could be regarded as heterogeneous, table 2 also show that Cochran's Q statistics for some (Executive function, $M$ and $M / F$ ) mean effects were significant, indicating that there is meaningful variance between the studies that are contributing to each mean.

Table2. Mean effects for impact of mindfulness on neurocognitive processing components,

\begin{tabular}{|c|c|c|c|c|c|c|c|c|c|}
\hline \multirow{2}{*}{ 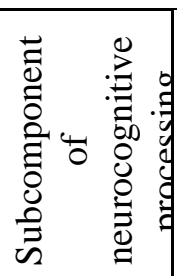 } & \multirow{2}{*}{ 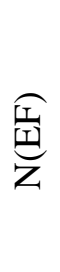 } & \multirow{2}{*}{ 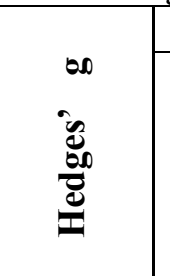 } & \multicolumn{2}{|c|}{$95 \% \mathrm{CIs}$} & \multicolumn{2}{|c|}{ Hetrogenity } & \multirow{2}{*}{ 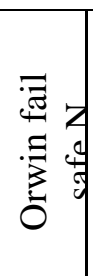 } & \multirow{2}{*}{$\begin{array}{l}n \\
0 \\
0 \\
0 \\
0 \\
0 \\
\infty \\
\infty \\
\infty\end{array}$} & \multirow{2}{*}{ 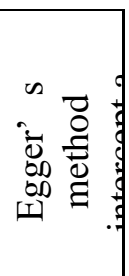 } \\
\hline & & & 离 & $\begin{array}{l}\overline{\grave{\Delta}} \\
\tilde{a}\end{array}$ & Q & I & & & \\
\hline $\begin{array}{c}\text { Executiv } \\
\mathrm{e} \\
\text { function }\end{array}$ & 13 & $\begin{array}{c}0.114 \\
(\mathrm{R})\end{array}$ & -0.15 & 0.38 & $41.43 * * *$ & 71.03 & \multirow{7}{*}{140} & \multirow{7}{*}{0.15} & \multirow{7}{*}{$1.57^{*}$} \\
\hline $\begin{array}{l}\text { Working } \\
\text { memory }\end{array}$ & 9 & $0.35 * *$ & 0.17 & 0.52 & 8.09 & 0.10 & & & \\
\hline $\begin{array}{l}\text { Attention } \\
\text { function }\end{array}$ & 6 & $0.32 * *$ & 0.14 & 0.51 & 7.43 & 32.74 & & & \\
\hline Overall & 28 & $0.31 * * *$ & 0.17 & 0.42 & 68.99 & $60.86 * * *$ & & & \\
\hline \multicolumn{7}{|c|}{ Gender } & & & \\
\hline $\mathrm{M} / \mathrm{F}$ & 16 & $0.25 * *(\mathrm{R})$ & 0.07 & 0.44 & $37.48^{* *}$ & 59.98 & & & \\
\hline $\mathrm{F}$ & 12 & $0.27(\mathrm{R})$ & -0.04 & 0.58 & $31.49 * *$ & 65.07 & & & \\
\hline
\end{tabular}

Note: $* * *<0001,{ }^{*} * \mathrm{p}<001,{ }^{*} \mathrm{p}<0.05, \mathrm{~N}(\mathrm{EF}), \mathrm{R}$ (Random), Number of effect sizes, Orwin fail safe $\mathrm{N}$ (Number of missing studies that would bring p-value to >alpha, M/F(Male/Female, $\mathrm{F}($ Female).

Testing for Publication Bias: The tests used to quantify publication bias (Begg's method kendall's Tau) suggested a presence of publication bias for cognitive function, but not significant in Egger's methods. Orwin's "Fail safe N" indicated that for cognitive function, 140 unpublished studies averaging null results would be needed to reduce the mean effect size value past 0.1 (respectively).

Publication bias based on funnel diagrams can be detected when the points are not symmetrically dispersed which can due to the very large values of the effect size and their very large standard error(Like the figure before the sensitivity analysis). As you can see in Figure 3, it was found that the 3 sizes of the effect have asymmetric and skewed values (effect sizes greater than the absolute value of 1), which made the graph asymmetric (figure 3.funnel plot before sensitivity analysis), In this study, after analyzing the sensitivity, we removed these 3 effect sizes, which have more homogeneous effect sizes than the first diagram (figure 3.funnel plot after sensitivity analysis), Figure3). Also, based on the statistical index (Fail-safe N) after entering 195 non-significant effect sizes in to the mea-analyze, the calculated composition effect sizes becomes non-significant (Figure3). 

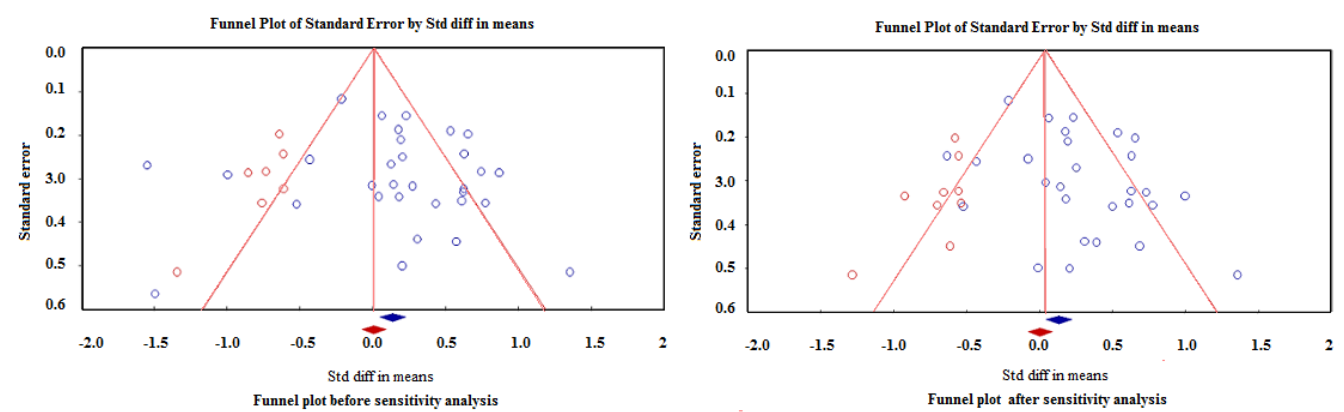

\section{Discussion}

This meta-analytic integration of 17 studies provides important and novel insights into mindfulness affects cognitive functions. Mindfulness can impact on both cognitive functions domain attention function and working memory, this result concordance with conclusions of review studies $(2,15,26)$. Consistent with Chiesa A, et al in 2015 finding of this study showed that mindfulness intervention had no effect on executive performance $(2,27)$. In order to justify the findings, as with self-reported memory, it is possible that patients' perceptions of processing speed difficulties will be influenced by anxiety and neuroticism. However, it is also possible that the accuracy of patients' perceived cognitive difficulties is domain dependent (28) and we can point out that Mindfulness Interventions (MIs) may cause changes in the brain that self-regulate emotions and that might be linked to raised cognitive reserve or recover cognitive functions in MS patients (29), and there is many researchers also speculate that the improvements in emotional stability may have implications for increasing cognitive function. For instance, studies have shown decreases in both perceived stress and cortisol levels after mindfulness $(15,30)$. Mindfulness training reduced participants' fatigue while anxiety ratings, fatigue and anxiety may be particularly critical in affecting information processing(31). Mindfulness is based on promoting a balance between a relaxed and vigilant state of mind(31). The ability to self-regulate emotions has been found to be a key component in enhancing cognition(32). As described before that cognitive slowing related to anxiety(33) and MI has been shown to positively influence on anxiety in MS patients(34) another finding of this study was to improve the detached mindfulness on anxiety symptoms. This finding is in line with the study applied detached mindfulness to treat depression and anxiety(3).

However, despite these acceptable mechanisms, at present there is incomplete evidence to confidently support the empirical link between mindfulness and executive function. This may be due to a lack of studies, rather than a null effect per se. More high-quality studies on the effects of mindfulness on executive function are imperative to build a substantive evidence base worthy of extrapolating so that health professionals can make confident decisions when prescribing mindfulness interventions. Perhaps more research will help us to draw conclusions and comment with more certainty.

\section{Conclusion}

There emerges to be proof that mindfulness improves cognitive function. In healthy adults, researches propose that short session of mindfulness improve attention function and working memory; however, there is adequate proof to emphasis alternation in cognitive functions after short- and long-term interventions Some evidence proofs mindfulness ability to promote perseveration in children and adolescents after short-term treatments, while improvements in working memory and mental flexibility have been reported in older adults. Short-term treatment researches have detected recovery in inhibitory control among impulsive prisoners and in working memory and attention among patients with type 2 diabetes mellitus. Most researches conducted 
among patients with multiple sclerosis did not suggest improvements in executive function. More studies should evaluate the efficacy of mindfulness effects on cognitive functions, especially in populations with cognitive deficits. Future studies should also separate mindfulness subcomponent effects on cognition function and investigate the neurophysiologic pathways of mindfulness-induced cognitive functions promote.

\section{Acknowledgments}

The authors express their appreciation to the Dr Javad Mesrabadi of Azarba Azarbaijan University of Shahid Maddani. This study is part of protocol was registered at Iranian registry of clinical trial (www.irct.ir; IRCT registration number: IRCT2016112728119N5)

\section{Funding}

This study is part of Ph.D thesis supported by Hamadan University of Medical Sciences (Grant NO: 9609286009).

\section{Conflict of interest}

All authors declare that have no conflict of interest.

\section{References}

1. Kabat-Zinn J. Mindfulness. Mindfulness. 2015;6(6):1481-3.

2. Chiesa A, Calati R, Serretti A. Does mindfulness training improve cognitive abilities? A systematic review of neuropsychological findings. Clinical psychology review. 2011;31(3):449-64.

3. Ahmadpanah M, Paghale SJ, Bakhtyari A, Kaikhavani S, Aghaei E, Nazaribadie M, et al. Effects of psychotherapy in combination with pharmacotherapy, when compared to pharmacotherapy only on blood pressure, depression, and anxiety in female patients with hypertension. Journal of health psychology. 2016;21(7):1216-27.

4. Ahmadpanah M, Nazaribadie M, Aghaei E, Ghaleiha A, Bakhtiari A, Haghighi M, et al. Influence of adjuvant detached mindfulness and stress management training compared to pharmacologic treatment in primiparae with postpartum depression. Archives of women's mental health. 2018;21(1):65-73.

5. Pollock JL, Cruz J. Contemporary theories of knowledge: Rowman \& Littlefield; 1999.

6. Lezak MD, Howieson DB, Loring DW, Fischer JS. Neuropsychological assessment: Oxford University Press, USA; 2004.

7. Chiesa A, Serretti A. Mindfulness based cognitive therapy for psychiatric disorders: a systematic review and meta-analysis. Psychiatry research. 2011;187(3):441-53.

8. Eberth J, Sedlmeier P. The effects of mindfulness meditation: a meta-analysis. Mindfulness. 2012;3(3):174-89.

9. Leyland A, Rowse G, Emerson L-M. Experimental effects of mindfulness inductions on selfregulation: Systematic review and meta-analysis. Emotion. 2019;19(1):108.

10. Chi X, Bo A, Liu T, Zhang P, Chi I. Effects of mindfulness-based stress reduction on depression in adolescents and young adults: a systematic review and meta-analysis. Frontiers in psychology. 2018;9:1034.

11. Zhang J, Xu R, Wang B, Wang J. Effects of mindfulness-based therapy for patients with breast cancer: a systematic review and meta-analysis. Complementary therapies in medicine. 2016;26:1-10. 
12. Song Y, Lindquist R. Effects of mindfulness-based stress reduction on depression, anxiety, stress and mindfulness in Korean nursing students. Nurse education today. 2015;35(1):8690.

13. Ramel W, Goldin PR, Carmona PE, McQuaid JR. The effects of mindfulness meditation on cognitive processes and affect in patients with past depression. Cognitive therapy and research. 2004;28(4):433-55.

14. Bamber MD, Morpeth E. Effects of mindfulness meditation on college student anxiety: A meta-analysis. Mindfulness. 2019;10(2):203-14.

15. Luu K, Hall PA. Hatha yoga and executive function: a systematic review. The Journal of Alternative and Complementary Medicine. 2016;22(2):125-33.

16. Blanck P, Perleth S, Heidenreich T, Kröger P, Ditzen B, Bents H, et al. Effects of mindfulness exercises as stand-alone intervention on symptoms of anxiety and depression: Systematic review and meta-analysis. Behaviour Research and Therapy. 2018;102:25-35.

17. Alfonso JP, Caracuel A, Delgado-Pastor LC, Verdejo-García A. Combined goal management training and mindfulness meditation improve executive functions and decisionmaking performance in abstinent polysubstance abusers. Drug and alcohol dependence. 2011;117(1):78-81.

18. Ching H-H, Koo M, Tsai T-H, Chen C-Y. Effects of a mindfulness meditation course on learning and cognitive performance among university students in Taiwan. Evidence-Based Complementary and Alternative Medicine. 2015;2015.

19. Johns SA, Von Ah D, Brown LF, Beck-Coon K, Talib TL, Alyea JM, et al. Randomized controlled pilot trial of mindfulness-based stress reduction for breast and colorectal cancer survivors: effects on cancer-related cognitive impairment. Journal of Cancer Survivorship. 2016;10(3):437-48.

20. Lam K, Seiden D. Effects of a Brief Mindfulness Curriculum on Self-reported Executive Functioning and Emotion Regulation in Hong Kong Adolescents. Mindfulness. 2020;11(3):627-42.

21. Luu K, Hall PA. Examining the acute effects of hatha yoga and mindfulness meditation on executive function and mood. Mindfulness. 2017;8(4):873-80.

22. Noone C, Hogan MJ. A randomised active-controlled trial to examine the effects of an online mindfulness intervention on executive control, critical thinking and key thinking dispositions in a university student sample. BMC psychology. 2018;6(1):13.

23. Jermann F, Van der Linden M, Gex-Fabry M, Guarin A, Kosel M, Bertschy G, et al. Cognitive functioning in patients remitted from recurrent depression: Comparison with acutely depressed patients and controls and follow-up of a mindfulness-based cognitive therapy trial. Cognitive Therapy and Research. 2013;37(5):1004-14.

24. Klainin-Yobas P, Kowitlawakul Y, Lopez V, Tang CT, Hoek KE, Gan GL, et al. The effects of mindfulness and health education programs on the emotional state and cognitive function of elderly individuals with mild cognitive impairment: a randomized controlled trial. Journal of Clinical Neuroscience. 2019;68:211-7.

25. Dunning DL, Griffiths K, Kuyken W, Crane C, Foulkes L, Parker J, et al. Research Review: The effects of mindfulness-based interventions on cognition and mental health in children and adolescents-a meta-analysis of randomized controlled trials. Journal of Child Psychology and Psychiatry. 2019;60(3):244-58.

26. Mrazek MD, Franklin MS, Phillips DT, Baird B, Schooler JW. Mindfulness training improves working memory capacity and GRE performance while reducing mind wandering. Psychological science. 2013;24(5):776-81.

27. Oken BS, Kishiyama S, Zajdel D, Bourdette D, Carlsen J, Haas M, et al. Randomized controlled trial of yoga and exercise in multiple sclerosis. Neurology. 2004;62(11):2058-64.

28. Roberg BL, Bruce JM, Lovelace CT, Lynch S. How patients with multiple sclerosis perceive cognitive slowing. The Clinical Neuropsychologist. 2012;26(8):1278-95.

29. Willekens B, Perrotta G, Cras P, Cools N. Into the Moment: Does Mindfulness Affect Biological Pathways in Multiple Sclerosis? Frontiers in behavioral neuroscience. 2018;12. 
30. Cohen DL, Wintering N, Tolles V, Townsend RR, Farrar JT, Galantino ML, et al. Cerebral blood flow effects of yoga training: preliminary evaluation of 4 cases. The Journal of Alternative and Complementary Medicine. 2009;15(1):9-14.

31. Zeidan F, Johnson SK, Diamond BJ, David Z, Goolkasian P. Mindfulness meditation improves cognition: Evidence of brief mental training. Consciousness and cognition. 2010;19(2):597-605.

32. Moore A, Malinowski P. Meditation, mindfulness and cognitive flexibility. Consciousness and cognition. 2009;18(1):176-86.

33. Rogers JM, Panegyres PK. Cognitive impairment in multiple sclerosis: evidence-based analysis and recommendations. Journal of Clinical Neuroscience. 2007;14(10):919-27.

34. Grossman P, Kappos L, Gensicke H, D'Souza M, Mohr DC, Penner IK, et al. MS quality of life, depression, and fatigue improve after mindfulness training: a randomized trial. Neurology. 2010;75(13):1141-9.

35. Chambers R, Lo BCY, Allen NB. The impact of intensive mindfulness training on attentional control, cognitive style, and affect. Cognitive therapy and research. 2008;32(3):303-22.

36. Flook L, Smalley SL, Kitil MJ, Galla BM, Kaiser-Greenland S, Locke J, et al. Effects of mindful awareness practices on executive functions in elementary school children. Journal of applied school psychology. 2010.

37. Quach D, Mano KEJ, Alexander K. A randomized controlled trial examining the effect of mindfulness meditation on working memory capacity in adolescents. Journal of Adolescent Health. 2016;58(5):489-96.

38. Shapero BG, Greenberg J, Mischoulon D, Pedrelli P, Meade K, Lazar SW. Mindfulnessbased cognitive therapy improves cognitive functioning and flexibility among individuals with elevated depressive symptoms. Mindfulness. 2018;9(5):1457-69.

39. Tang R, Broderick PC, Bono T, Dvoráková K, Braver TS. A College First-Year Mindfulness Seminar to Enhance Psychological Well-Being and Cognitive Function. Journal of Student Affairs Research and Practice. 2020:1-15.

40. Thierry KL, Vincent RL, Bryant HL, Kinder MB, Wise CL. A self-oriented mindfulnessbased curriculum improves prekindergarten students' executive functions. Mindfulness. 2018;9(5):1443-56.

41. Valls-Serrano C, Caracuel A, Verdejo-Garcia A. Goal Management Training and Mindfulness Meditation improve executive functions and transfer to ecological tasks of daily life in polysubstance users enrolled in therapeutic community treatment. Drug and Alcohol Dependence. 2016;165:9-14.

42. Wahbeh H, Goodrich E, Oken BS. Internet mindfulness meditation for cognition and mood in older adults: A pilot study. Alternative therapies in health and medicine. 2016;22(2):44.

43. Wood L, Roach AT, Kearney MA, Zabek F. Enhancing executive function skills in preschoolers through a mindfulness-based intervention: A randomized, controlled pilot study. Psychology in the Schools. 2018;55(6):644-60. 